\title{
Transient Expression of Laminin in the Optic Nerve of the Developing Rat
}

\author{
Steven C. McLoon, ${ }^{1}$ Linda K. McLoon, ${ }^{2}$ Sally L. Palm, ${ }^{3}$ and Leo T. Furcht ${ }^{3}$ \\ Departments of ${ }^{1}$ Cell Biology and Neuroanatomy, ${ }^{2}$ Ophthalmology, and ${ }^{3}$ Laboratory Medicine and Pathology, University of \\ Minnesota, Minneapolis, Minnesota 55455
}

The optic nerve of the developing rat was examined for the presence of laminin, an adhesive glycoprotein, to assess whether it might serve as a substrate for retinal axon growth in vivo. The optic stalk and nerve of developing rats were screened immunohistochemically for the presence of laminin before, during, and after the period of retinal axon growth. On embryonic day 14 (E14), laminin immunoreactivity was present in the ventral portion of the optic stalk, the same region in which the first retinal axons grow. Between E16 and postnatal day $10(P 10)$, cells positive for laminin were distributed throughout the cross-sectional area of the nerve. There was a progressive appearance of glial fibrillary acidic protein (GFAP) immunoreactivity, a marker for astrocytes, from the optic nerve head towards the chiasm beginning on E20. At the advancing front of GFAP immunoreactivity, cells were positive for both laminin and GFAP. Behind the front, laminin immunoreactivity disappeared from the cells. By P12, the only laminin immunoreactivity that remained within the optic nerve surrounded the vasculature. This is a time after the last retinal axons grow through the optic nerve.

Monolayer cell cultures were prepared from perinatal rat optic nerves and processed for immunohistochemistry to determine which astrocyte type was laminin-positive. Type 1 astrocytes, which primarily compose the immature nerve, are GFAP-positive, A2B5-negative, and laminin-positive. Type 2 astrocytes, a major component of the mature optic nerve, were GFAP-positive, A2B5-positive and laminin-negative.

An extract of developing optic nerve was analyzed by immunoblot along with laminin purified from Engelbreth-HolmSwarm (EHS) sarcoma. Purified laminin ran with SDS-PAGE under reducing conditions as 2 bands with $M_{r} s$ of 200,000 and 400,000 . Both bands reacted with antibodies to laminin. A low-salt extraction of whole optic nerve from $E 18$ rats resulted in 2 bands with the same $M_{r}$ as seen with laminin from EHS sarcoma. When only the inside of the optic nerve (which lacked the basal lamina and meninges that surround the outside) was processed, there was a dark 200,000 D band, but the $400,000 \mathrm{D}$ band was virtually absent.

\footnotetext{
Received June 3, 1987; revised Oct. 9, 1987; accepted Oct. 19, 1987

Technical assistance was rendered by Ms. Ruth Wilson and Ms. Lenore Mottaz We thank Dr. Doris Dahl for supplying the antisera to glial fibrillary acidic protein We thank Mr. Tom White and Dr. David Hamilton for advice and assistance with the Western blots. This work was supported by Grants EY05371, EY05372, and EY05432 from the National Institutes of Health, BRSG funds from the University of Minnesota, and a grant from Research to Prevent Blindness, Inc.

Correspondence should be addressed to Steven C. McLoon, Department of Cel Biology and Neuroanatomy, University of Minnesota, 4-135 Jackson Hall, 321 Church Street S.E., Minneapolis, MN 55455.

Copyright (C) 1988 Society for Neuroscience $0270-6474 / 88 / 061981-10 \$ 02.00 / 0$
}

These results are consistent with the hypothesis that laminin, or a variant form of laminin, serves as a substrate for retinal axon growth in the developing rat optic nerve.

Axons in the developing central nervous system grow along unique and highly stereotyped courses, often through a complex cellular terrain. Growing axons require a substrate to which they can adhere in order to extend (Letourneau, 1975a). Evidence from studies done in tissue culture suggests that growing axons can detect substrates of differing adhesivity, and, when given a choice, will selectively grow on the substrate to which they are most adherent (Letourneau, 1975b; Bonhoeffer and Huf, 1980; Noble et al., 1984; Hammarback et al., 1985). One possible explanation for the unique course taken by an axon in the developing brain is that a particular molecule may have a restricted distribution within the brain, and the growing axon could selectively adhere to and follow the path of this molecule. Certain glycoprotein and proteoglycan components of the extracellular matrix have been implicated in axon-substrate adhesion (Sanes, 1983). However, few candidates for axon-substrate molecules have been definitively identified in the developing central nervous system.

Laminin has unique binding characteristics that make it an appealing candidate for such a substrate molecule. It is a highmolecular-weight adhesive glycoprotein found in basal laminae (Timpl et al., 1979). It is also found outside traditional basal laminae in association with several cell types during development (Liesi, 1985; Rogers et al., 1986). Laminin has binding sites for glycosaminoglycans, collagen type IV, and certain cell surfaces (Terranova et al., 1983). Thus, laminin could mediate the attachment of a growth cone to other cells or structural components of the extracellular matrix. Laminin has been shown to be a component of neurite outgrowth-promoting factors found in conditioned media derived from a number of cell types (Davis et al., 1985; Lander et al., 1985).

Evidence obtained in tissue culture suggests that laminin is a suitable substrate molecule for the growth of retinal axons (Manthorpe et al., 1983; Rogers et al., 1983; Smalheiser et al., 1984). Laminin bound to the substratum promoted neurite outgrowth from retinal explants and dispersed retinal cell cultures. This activity was blocked by the addition of antibodies to laminin. Laminin was a more potent promoter of retinal neurite outgrowth than fibronectin, collagen (types I or IV), or various proteoglycans. Despite these intriguing data, laminin has not yet been identified along the path of growing optic axons in the developing central nervous system. In the study described here, the optic stalk or nerve before, during, and after the period in 
which retinal axons are growing was examined for the presence of laminin.

\section{Materials and Methods}

Tissue preparation. Black hooded rats, at $2 \mathrm{~d}$ intervals between embryonic day 14 (E14) (birth on day 22) and postnatal day 20 (P20) were anesthetized and perfused with $4 \%$ paraformaldehyde. The optic stalks or nerves of these animals were cryoprotected in $20 \%$ sucrose/phosphate buffer overnight, embedded in a homogenate of brain, and sectioned at $12 \mu \mathrm{m}$ in a cryostat. The sections were mounted on microscope slides and processed for immunohistochemistry.

Glial cell cultures. Monolayer cultures of optic nerve glia were prepared by a technique adapted from Raff(Raff et al., 1983b). Optic nerves were dissected from Black hooded rats on P3 or P7. The nerves were minced in Eagle's minimum essential medium (MEM) containing 0.02 M HEPES buffer. The tissue was then incubated in 4 changes of $0.02 \%$ collagenase and $0.25 \%$ trypsin in MEM over $1 \mathrm{hr}$. Next, the tissue was triturated in Dulbecco's MEM (from Gibco) containing 10\% heat-inactivated fetal calf serum (from Gibco), centrifuged at $500 \times g$ for 10 min, and plated onto polylysine-coated plastic tissue culture dishes in the same medium. The cultures were maintained at $37^{\circ} \mathrm{C}$ and $5 \% \mathrm{CO}_{2}$. The medium was changed daily. The cultures were processed for immunohistochemistry after 3 or $6 \mathrm{~d}$ in vivo.

Immunohistochemistry. Cultures were incubated for $2 \mathrm{hr}$ in ascites fluid containing A2B5 monoclonal antibodies (Eisenbarth et al., 1979) diluted 1:20 in PBS. After rinsing, they were fixed overnight in 4\% paraformaldehyde in phosphate buffer.

Cultures and sections were rinsed in PBS and treated with 10\% normal goat serum. They were then incubated overnight at $4^{\circ} \mathrm{C}$ in affinitypurified rabbit antiserum to laminin diluted 1:100 in PBS containing $0.3 \%$ Triton $\mathrm{X}-100$. The antiserum was produced in rabbit against laminin purified from mouse Engelbreth-Holm-Swarm (EHS) sarcoma (Palm and Furcht, 1983). The affinity-purified antiserum was shown not to cross-react with type IV collagen or other components of basement membrane. The cultures and sections were rinsed in PBS and then incubated for $1 \mathrm{hr}$ in goat anti-rabbit $\mathrm{IgG}$ conjugated to fluorescein isothiocyanate (FITC; Cappel). This incubation medium for the cultures also contained goat anti-mouse $\operatorname{IgG}$ conjugated to rhodamine isothiocyanate (RITC; Cappel). Sections and cultures were examined with a microscope equipped for epifluorescence. Cells were photographed with filters appropriate for distinguishing rhodamine or fluorescein fluorescence. The position of the photographed cells was plotted on paper by means of an X-Y plotter interfaced with the microscope stage.

All antibodies were then eluted from the sections and cultures by incubation in a solution of $0.1 \% \mathrm{KMnO}_{4}$ and $0.2 \% \mathrm{H}_{2} \mathrm{SO}_{4}$ for $2 \mathrm{~min}$, followed by $15 \mathrm{sec}$ in $0.5 \% \mathrm{Na}_{2} \mathrm{~S}_{2} \mathrm{O}_{5}$ (Tramu et al., 1978). To verify the removal of the antibodies, they were incubated in goat anti-rabbit immunoglobulins conjugated to FITC, rinsed, and examined with fluorescence microscopy. The sections and cultures were next incubated overnight in rabbit antiserum to glial fibrillary acidic protein (GFAP) (Dahl and Bignami, 1976) diluted $1: 1000$ in PBS containing 0.3\% Triton $\mathrm{X}-100$. They were rinsed in PBS, incubated for $1 \mathrm{hr}$ in goat anti-rabbit $\mathrm{IgG}$ conjugated to fluorescein, and again examined with fluorescence microscopy. Cells that had been previously photographed were located with the aid of the $X-Y$ plotter and rephotographed. Selected sections were counterstained with $0.01 \%$ ethidium bromide to show all cells.

Control sections of optic nerve from each age were incubated in preimmune serum or primary antisera absorbed with laminin in place of the primary antibodies, and processed as described above. No fluorescence significantly above background levels was found within these sections.

Control cultures were processed with one of the 3 primary antibodies replaced by normal serum, preimmune serum, or antiserum absorbed with the antigen. The controls indicated that a faint red fluorescence with the filters for FITC resulted from RITC labeling of the A2B5 antibody. The color difference between FITC and RITC was obvious to the eye, but combined with the spectral sensitivity of the film, it caused difficulties with black and white photography. No cross-reactivity of the secondary antibodies was observed.

Western blots. Optic nerves (30-60/gel) were dissected from anesthetized $\mathrm{E} 18$ rats. The internal contents of half the nerves were obtained by massaging along the length of the nerves with fine forceps. The internal contents of the nerves lacked the basal lamina or meninges surrounding the outside of the nerve, which was verified with immu- nohistochemistry. The optic nerve samples were homogenized in $3.4 \mathrm{M}$ $\mathrm{NaCl} / 0.05 \mathrm{M}$ Tris $/ 0.14 \mathrm{~mm} p$-hydroxymercuribenzoate $/ 0.3 \mathrm{~mm}$ phenylmethanesulfonyl fluoride. The homogenate was centrifuged at $7000 \times$ $g$ for $10 \mathrm{~min}$, and the supernatant was discarded. The pellet was resuspended and homogenized in the same buffer, but containing only 0.5 $\mathrm{M} \mathrm{NaCl}$. This was again centrifuged as above. The supernatant was dialyzed against $0.05 \mathrm{M}$ Tris buffer with protease inhibitors (as above) and then lyophilized. Samples were resuspended in sample buffer that contained 3 or $6 \% 2$-mercaptoethanol, boilcd for $5 \mathrm{~min}$, and run on a $5 \%$ SDS-PAGE (Laemmli, 1970). The proteins were transferred from the polyacrylamide gel to nitrocellulose electrophoretically (Towbin et al., 1979). Strips of the blots were blocked with $10 \%$ BSA/PBS, incubated overnight in antiserum to laminin (as above), rinsed in PBS, and incubated in goat anti-rabbit IgG conjugated to HRP (from Sigma Chemical Co.) at 1:1000 for $2 \mathrm{hr}$. After rinsing in PBS, the blot strips were incubated in diaminobenzidine and hydrogen peroxide. Laminin purified from EHS sarcoma was run in a parallel lane and processed identically. A mixture of proteins (Pharmacia) with known molecular weights from 67 to $669 \mathrm{kDa}$ were run in a parallel lane of several gels. This lane of the blot was stained to show all proteins with colloidal gold (Aurodye; Janssen Pharmaceutica, used according to the manufacturer's instructions).

\section{Results}

\section{Optic nerve immunohistochemistry}

The optic stalk or nerve of rats at different ages of development was screened for the presence of laminin by immunohistochemistry. Laminin immunoreactivity was present in the optic stalk from E14 embryos (Fig. 1A). The cells in the optic stalk at this age are radially arranged, running from the lumen to the external surface of the stalk. The staining was heavy towards the lumen of the stalk, but also appeared to outline the cells up to the external limiting membrane. The most intense staining was on the cells in the ventral portion of the stalk. Some laminin-immunoreactive cells were also present in the dorsal portion of the stalk, but the brightness of the staining on individual cells was always significantly less than that seen in the ventral portion.

As axons grew into the stalk, beginning on E14, the optic nerve was formed. By E16 most cells of the optic nerve had lost their radial orientation. Between E16 and P10, cells that stained with the laminin antibody were distributed throughout the crosssectional area of the optic nerve. There continued to be an uneven distribution of the immunoreactive cells. Particularly at the early ages, the highest density of laminin-positive cells and the brightest-staining cells were concentrated in a crescent centered on the ventral aspect of the nerve (Fig. $1 B$ ). In cross section of the optic nerve, the laminin-positive cells were typically stellate-shaped (Fig. 2). In longitudinal sections of the optic nerve, the stellate shape of the cells was less obvious. The laminin-positive cclls appcared to form linear arrays oriented along the length of the nerve (Fig. 3). By through-focusing on slightly tangential sections near the surface of the nerve, it became apparent that some laminin-positive cells contributed a process to the external limiting membrane. This process expanded into an endfoot that appeared to be laminin-positive on its external surface.

At birth (E22 or P0), the first blood vessels could be distinguished in the optic nerve. The blood vessels were lamininpositive, as were cells not associated with blood vessels (Fig. $1 C)$. There was a progressive loss of the cellular staining for laminin, which began at the eye and progressed towards the brain. The loss of laminin immunoreactivity was first apparent near the eye at P0. By P12, the only laminin reactivity that remained within the optic nerve surrounded the vasculature (Fig. 1D). At this and all older ages, including adults (McLoon, 

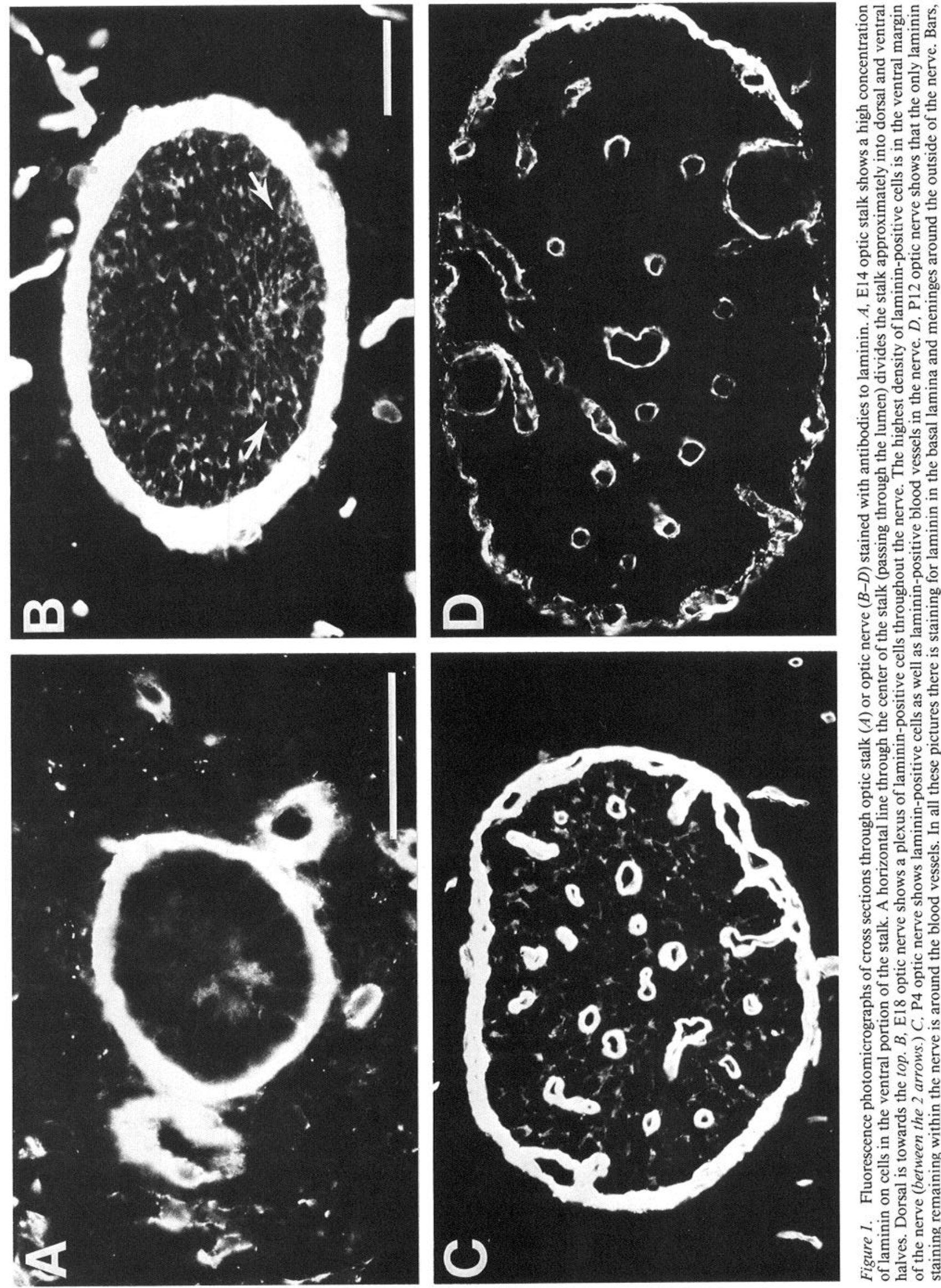

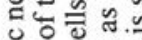

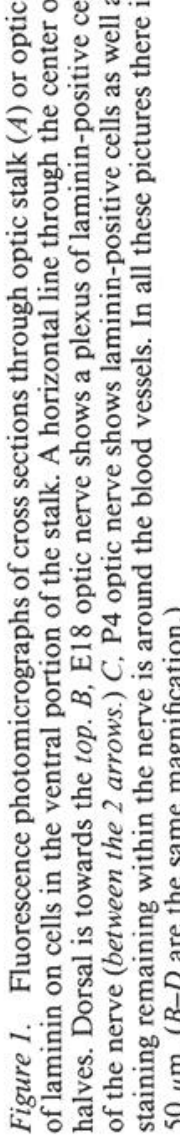




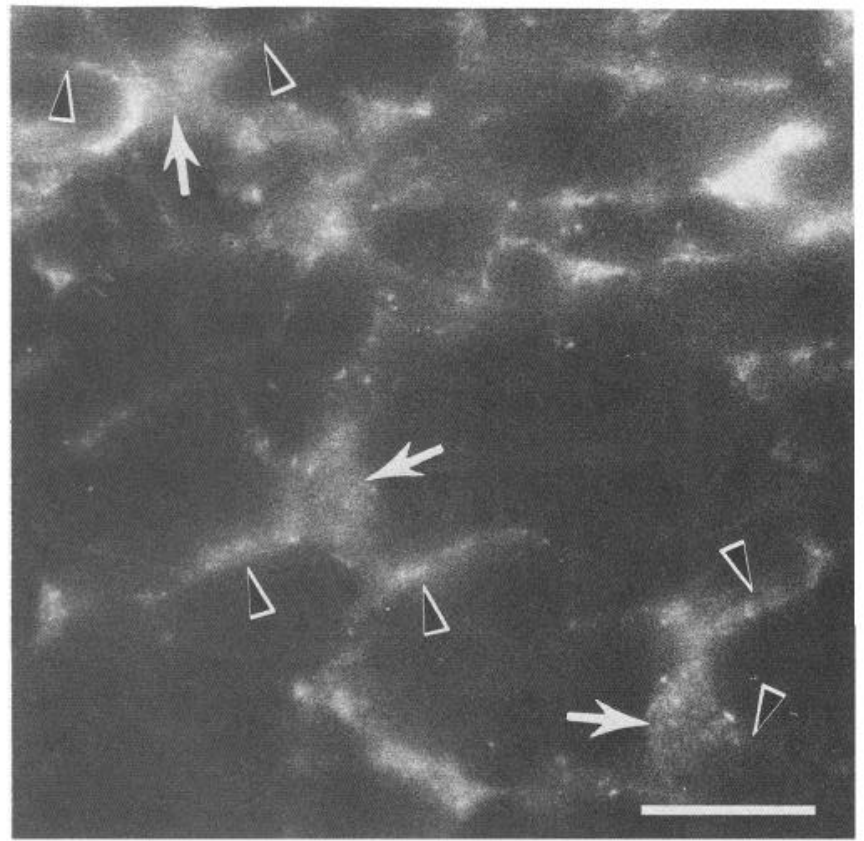

Figure 2. High-power fluorescence photomicrograph of a cross section of E18 optic nerve that shows that the laminin staining within the nerve is associated with a stellate-shaped cell. The somas of 3 cells are marked with arrows, and several processes of the cells are indicated by open arrowheads. Bar, $10 \mu \mathrm{m}$.

1986), the cellular staining for laminin was no longer visible. This was true even when the primary antibody was 10 times more concentrated than the dilution used for most of this study.

The meninges and basal lamina surrounding the optic nerves were positive for laminin at all ages. The laminin staining around the outside of the nerve and that associated with the vasculature was always of significantly greater intensity than that of the cells within the nerve.

The laminin antibodies were eluted from the sections, and the sections were restained with antibodies to GFAP. Identified cells that were laminin-positive were examined for GFAP immunoreactivity. No GFAP-positive cells were encountered on E18 within the rat optic nerve. On E20, cells in the optic nerve near the eye were positive for GFAP. Many of the cells that were positive for GFAP were also positive for laminin (Fig. 4). Although no attempt was made at quantitation, it was our impression that the majority, if not all, GFAP-positive cells at this age were also laminin-positive. There was a progressive appearance of GFAP-positive cells in the optic nerve from the eye towards the chiasm over the next 8 days of development. In longitudinal sections of the nerve during this period, it was apparent that laminin and GFAP coexisted in cells in only a short segment of the nerve at any single time point. Towards the eye from this segment, the GFAP-positive cells were for the most part laminin-negative. Towards the chiasm from this segment, laminin-positive cells were present, but there were no GFAP-positive cells.

\section{Glial cell culture immunohistochemistry}

Cultures of astrocytes from perinatal optic nerve were stained with a number of antibodies in order to determine the type or types of astrocytes that were laminin-positive. Also, single cells can be much more clearly identified in cell culture than in tissue

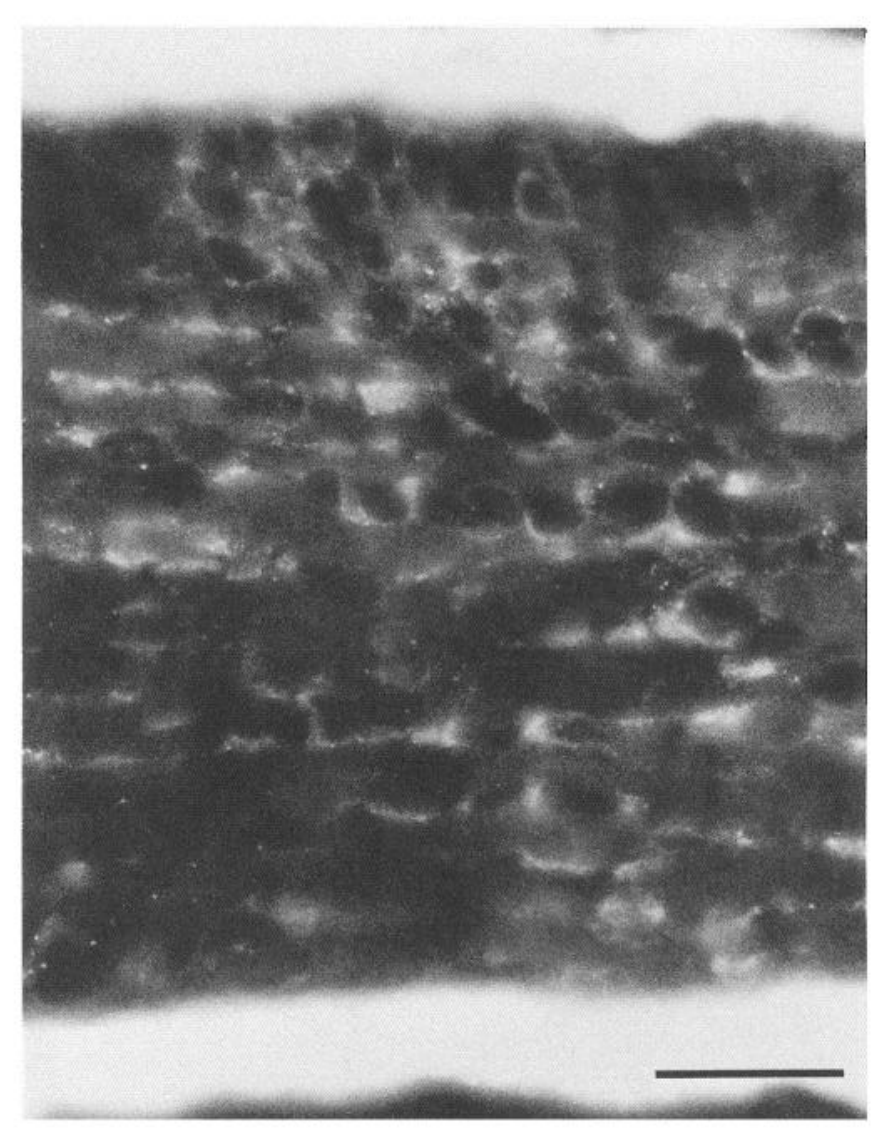

Figure 3. Fluorescence photomicrograph of a longitudinal section of E18 optic nerve that shows the laminin-positive cells arranged in linear arrays oriented along the long axis of the nerve (right to left). The outside of the nerve is at the top and bottom. Bar, $25 \mu \mathrm{m}$.

sections, allowing a more definitive determination of the coexistence of laminin and GFAP in cells. Astrocytes, defined by positive GFAP immunoreactivity and cultured for $3 \mathrm{~d}$ from P7 rat optic nerve, were principally of 2 types. The first cell type had large somas with considerable cytoplasm, was round or polygonal in shape, and seldom had more than 2 processes, if any (Fig. $5 A$ ). These cells did not stain with the A2B5 monoclonal antibody (Fig. $5 B$ ). Approximately $80 \%$ of the GFAPpositive cells were of this type. The second GFAP-positive cell type had little cytoplasm, was stellate in shape, and usually had several long processes (Fig. 6A). This cell type was A2B5-positive (Fig. $6 \mathrm{~B}$ ). The A2B5 staining appeared as bright punctate staining on the soma and very faint diffuse staining on the processes. Of the GFAP-positive cells, $19 \%$ were of this type. These appear to correspond to previous descriptions of types 1 and 2 astrocytes, respectively (Raff et al., 1983a). There were minor differences in the degree of differentiation and the proportions of the 2 types of astrocytes, depending on whether P3 or P7 optic nerve was used, or whether they were maintained in culture for 3 or $6 \mathrm{~d}$.

Types 1 and 2 astrocytes exhibited considerably different staining patterns with antibodies to laminin. Type 1 astrocytes showed intense staining for laminin. After $3 \mathrm{~d}$ in culture, the staining was bright intracellularly, with a small amount of punctate staining on the cell surface (Fig. 5C). After $6 \mathrm{~d}$ in culture, there was a significant increase in the punctate staining on the cell surface. Several living cultures were stained with the laminin 

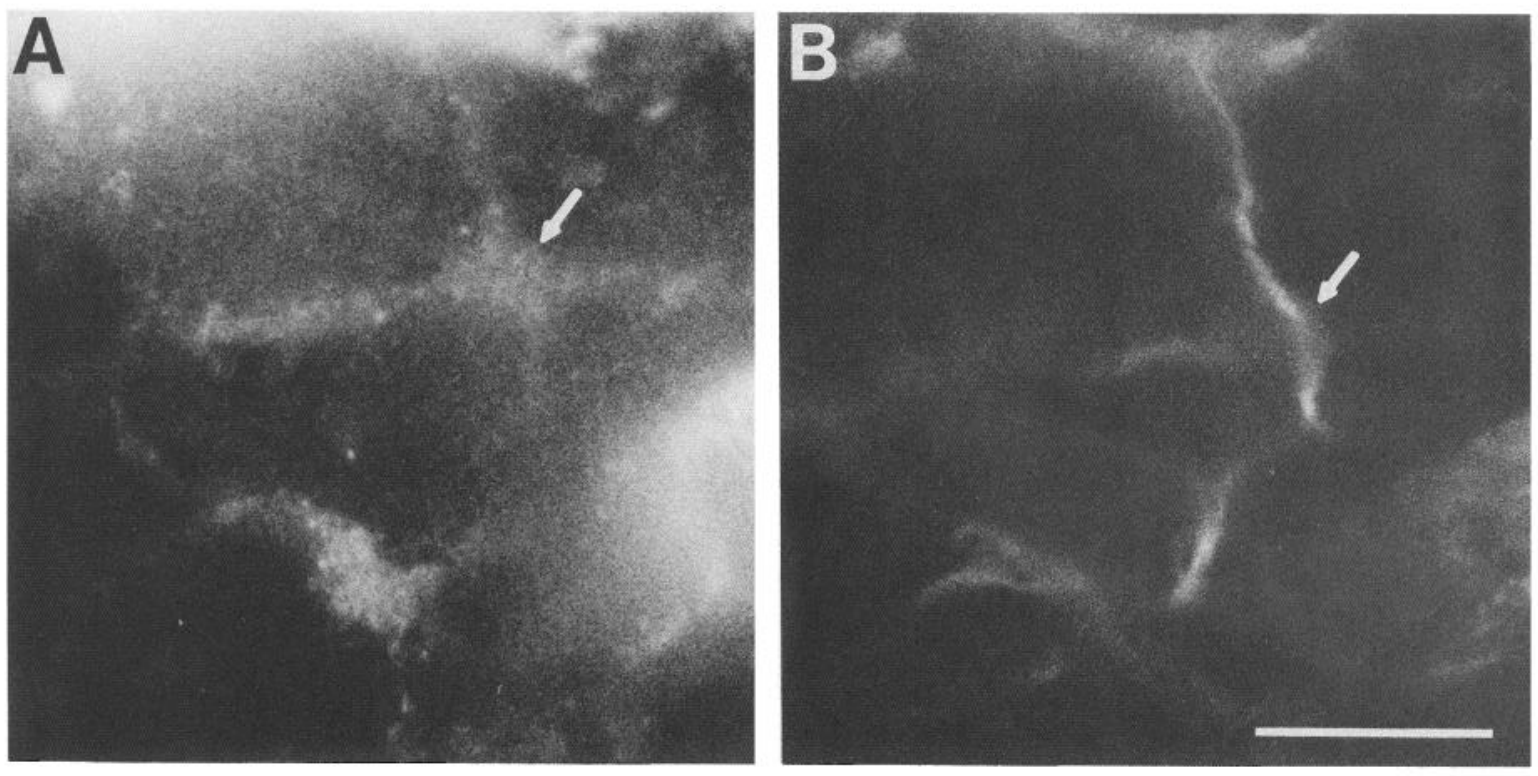

Figure 4. Fluorescence photomicrographs of a section of P0 optic nerve first stained with anti-laminin antibodies $(A)$, and, after the first antibodies were eluted, restained with antibodies to GFAP $(B)$. The same cells and processes appear to be both laminin- and GFAP-positive. Arrows indicate comparable structures in each picture. A blood vessel is in the lower right corner. The outside of the nerve is to the top. A slightly different plane of focus was used in the 2 pictures in order to emphasize the intracellular GFAP and the cell surface laminin. Bar, $10 \mu \mathrm{m}$.

antibody (not shown). Cells with the morphology of type 1 astrocytes were still positive for laminin. Since the antibody was applied to living cells not permeabilized by fixative or detergent, the laminin antibody appears to have reacted with laminin on the external surface of the cells. (This group of cultures was not tested with the A2B5 antibody.) Type 2 astrocytes did not stain with the laminin antibody (Fig. 6C). Characteristics of type 1 and type 2 astrocytes are summarized in Table 1.

Other cell types encountered did not fit the pattern of staining described above. One of these was a polygonal-shaped cell with short processes. These cells stained positive for GFAP, A2B5, and laminin. They accounted for approximately $1 \%$ of the GFAPpositive cells. Another cell type that did not fit the above pattern was a process-bearing cell somewhat like the type 2 astrocyte. They were positive for A2B5 but negative for GFAP and laminin. Finally, there were cells that were positive for laminin but negative for A2B5 and GFAP. No effort was made to characterize these cells further.

\section{Western blot analysis of optic nerve}

Western blot analysis of E18 rat optic nerve was undertaken to help determine whether the laminin immunoreactivity indicated the presence of laminin in the developing optic nerve. E18 nerve was chosen because immunohistochemistry revealed a large number of laminin-positive cells within the nerve at this age, and it precedes the appearance of laminin-positive blood vessels within the nerve. Laminin purified from EHS sarcoma, run on an SDS polyacrylamide gel under reducing conditions, transblotted to nitrocellulose, and stained with an antibody to laminin resulted in 2 bands at approximately 200 and $400 \mathrm{kDa}$ (Fig. 7A). A low-salt extraction of whole optic nerve gave 2 bands that were indistinguishable from those of EHS sarcoma laminin (Fig. $7 B$ ). It is possible, however, that these bands represent only the laminin present in the basal lamina and meninges surrounding the outside of the optic nerve. An extract of the inside of the optic nerve was prepared that, as shown by im- munohistochemistry, was free of meninges or organized basal lamina. A Western blot analysis of a low-salt extraction of the inside of the nerve revealed a dark $200 \mathrm{kDa}$ band with little or no $400 \mathrm{kDa}$ band (Fig. $7 \mathrm{C}$ ). The remaining outside of the nerve gave both the 200 and $400 \mathrm{kDa}$ bands when processed in the same manner (not shown).

\section{Discussion}

Immunohistochemistry revealed the transient expression of laminin in the developing rat optic stalk and nerve. Laminin immunoreactivity was present in the optic stalk on E14, the earliest age examined, and remained on cells within the optic nerve through P10. As early as E20, cells were found in the optic nerve that were positive for laminin and GFAP, a marker for astrocytes. In cultures prepared from neonatal optic nerve, type 1 astrocytes were found to be laminin-positive, whereas type 2 astrocytes were laminin-negative. Western blot analysis of an extract of whole E18 optic nerve run on an SDS-polyacrylamide gel under reducing conditions revealed 200 and 400 $\mathrm{kDa}$ bands. Whole optic nerve included the basal lamina surrounding the outside of the nerve. When the inside of the nerve alone was processed under the same conditions, a dark $200 \mathrm{kDa}$ band was present, while the $400 \mathrm{kDa}$ band was significantly reduced or absent.

Table 1. Astrocyte characteristics ${ }^{a}$

\begin{tabular}{llrlll} 
& Shape & Percentage $^{b}$ & GFAP & A2B5 & Laminin \\
\hline Type 1 & Polygonal & $80 \pm 3$ & + & - & + \\
Type 2 & Stellate & $19 \pm 4$ & + & + & - \\
Other & Polygonal & $1 \pm 1$ & + & + & +
\end{tabular}

${ }^{a}$ Cells obtained from P7 optic nerve after $3 \mathrm{~d}$ in culture. One hundred astrocytes (defined as GFAP-positive) were counted in each of 10 dishes. All astrocytes (up to a total of 100 in a given dish) in randomly selected microscope fields were counted and classified.

${ }^{b}$ Mean percentage of all GFAP-positive cells \pm SD. 
1986 McLoon et al. • Laminin in the Developing Rat Optic Nerve
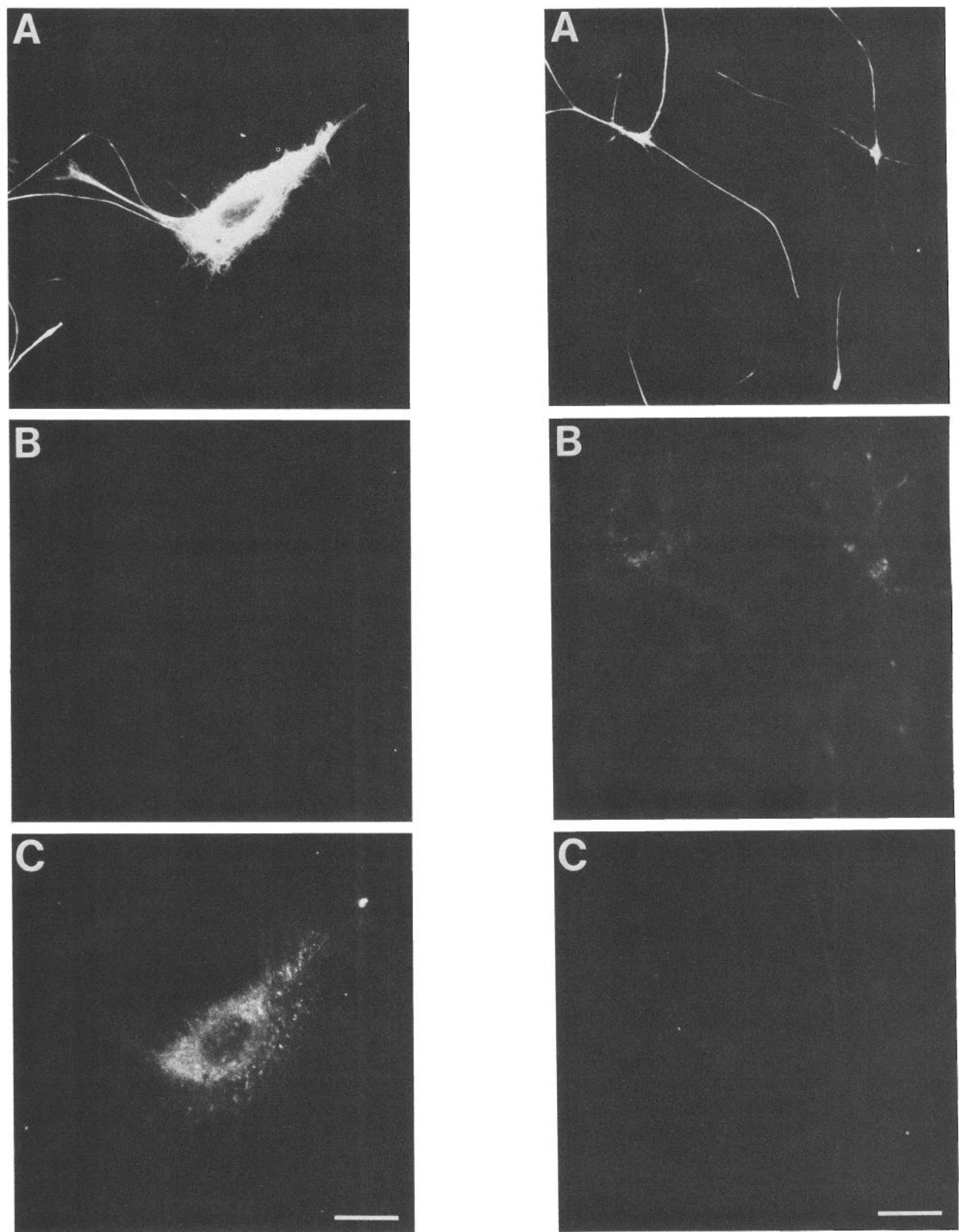

Figure 5. Fluorescence photomicrographs of a type 1 astrocyte obtained from $\mathrm{P} 7$ optic nerve after $3 \mathrm{~d}$ in culture. This cell is $(A)$ positive for GFAP, $(B)$ negative for A2B5, and $(C)$ positive for laminin. The process in the lower left corner is from a cell that is A2B5-positive and laminin-negative. Bar, $20 \mu \mathrm{m}$.

Figure 6. Fluorescence photomicrographs of 2 type 2 astrocytes from the same culture used for the cell in Figure 5. These cells are $(A)$ positive for GFAP and $(B)$ A2B5, and $(C)$ negative for laminin. (The faint fluorescence at the FITC wavelength in $C$ is due to a bleedthrough of the red RITC fluorescence in B.) Bar, $20 \mu \mathrm{m}$. 
Laminin purified from a number of sources, including EHS sarcoma (Timpl et al., 1979), myoblasts (Kuhl et al., 1982), endodermal carcinoma (Sakashita et al., 1980), yolk sac tumor, and amniotic membrane (Albrechtsen et al., 1981), gives 200 $\mathrm{kDa}$ bands, the $\mathrm{B}_{1}$ and $\mathrm{B}_{2}$ chains, and a $400 \mathrm{kDa}$ band, the $\mathrm{A}$ chain, with SDS-PAGE run under reducing conditions. The virtual absence of the $400 \mathrm{kDa}$ or A subunit of laminin within the optic nerve could be explained in a number of ways. This subunit could be complexed to other molecules so as to block binding by the antibodies or prevent extraction by the low-salt buffer. It is also possible that the molecule produced in the developing optic nerve only has some homologies with laminin found in basal lamina. It may lack a subunit or have an altered subunit with different antibody-binding characteristics. Conditioned media from Schwann cells and Schwannoma cells contain a laminin-like molecule that also appears to lack the A chain (Cornbrooks et al., 1983; Palm and Furcht, 1983; Davis et al., 1985; McLoon and McLoon, 1988). Electron microscopy suggests that laminin from Schwannoma cells lacks one of the 3 short arms seen with laminin obtained from basal lamina (Davis et al., 1985). This suggests that the $400 \mathrm{kDa}$ subunit is not present in these molecules. There is some precedent for differential synthesis of the $B_{1}, B_{2}$, and $A$ subunits of laminin (Cooper and MacQueen, 1983). It was reported that mouse oocytes made only the $B_{1}$ chain of laminin, while 4-8-cell embryos made $B_{1}$ and $B_{2}$ chains. Production of the $A$ chain did not appear until the 16-cell stage. One possible explanation is that, within the developing optic nerve, only B-type chains are synthesized and used to make laminin. Two B-type chains could associate into a 2-arm structure if the alpha helices did not require the central core $\mathrm{A}$ chain to make the long arm. Another possibility is that only half of the A chain is expressed, resulting in a laminin made up of three $200 \mathrm{kDa}$ subunits. It may be functionally important that a variant form of laminin is used during development. Laminin that lacks a particular subunit might be less tightly cross-linked into a matrix so as to facilitate its degradation when its usefulness is ended.

Several cell types or developmental stages of a single cell type appear to produce laminin in the developing optic nerve. The cells that compose the early optic nerve are undifferentiated and appear to be relatively homogeneous. Many, if not all, cells in the optic nerve up until P0 appear to be laminin-positive. Previous studies have shown that astrocytes from some developing brain regions or from the mature injured brain can synthesize and secrete laminin (Liesi et al., 1983, 1984). GFAP is a marker specific for astrocytes (Bignami et al., 1972; Schachner et al., 1977). The first GFAP immunoreactivity appears in the nerve at E20 near the optic nerve head, and there is a progressive appearance of GFAP-positive cells towards the chiasm. Doublestaining with antibodies for laminin and GFAP shows a brief developmental period for any given segment of the nerve in which the 2 molecules are colocalized in cells. This finding suggests that a stage in the differentiation of at least some astrocytes involves laminin synthesis.

Astrocytes in the optic nerve can be further characterized into 2 types of astrocytes to synthesize laminin further emphasizes 1 astrocytes were positive for laminin, while the type 2 astrocytes were negative for laminin. The difference in the abilities of the 2 types of astrocytes to synthesize laminin further emphasizes the difference between the 2 cell types. A third cell type that did not fit this pattern was identified. These cells had the shape of type 1 astrocytes and were positive for GFAP, A2B5, and lam-

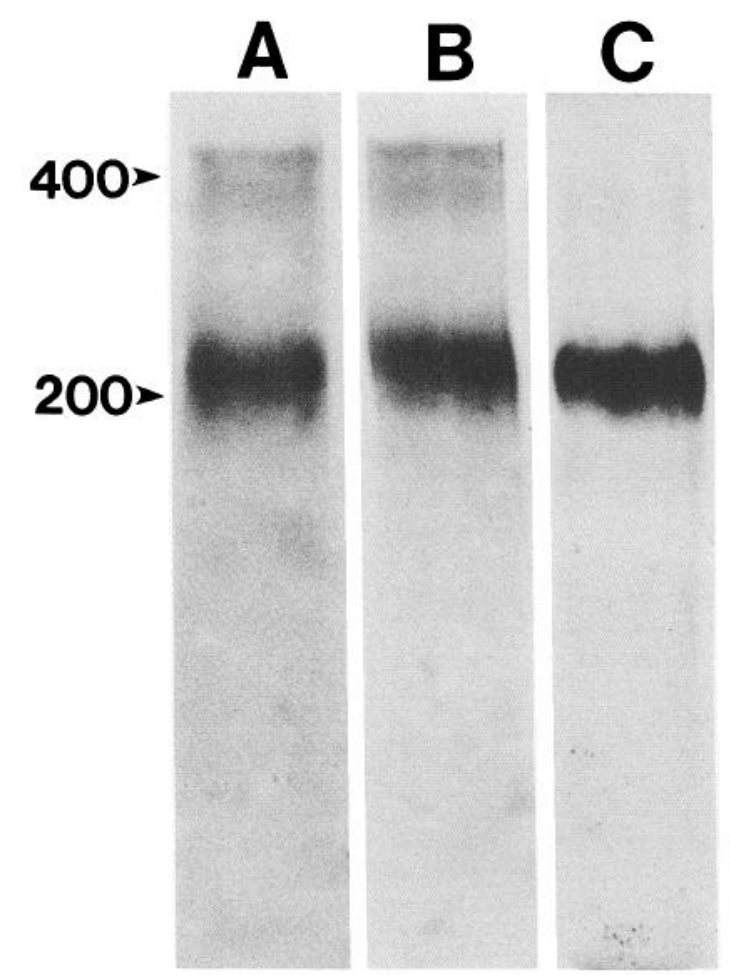

Figure 7. Immunoblot after SDS-PAGE of $(A)$ laminin purified from EHS sarcoma, $(B)$ a low-salt extraction of whole E18 optic nerve, and (C) a low-salt extraction of the inside of E18 optic nerve (which lacks the meninges and associated basal lamina). All lanes were stained with antibodies to laminin. The 3 lanes are nonconsecutive lanes of the same gel. The 2 bands of the purified laminin were used as molecular-weight standards of 400 and $200 \mathrm{kDa}$, which had been confirmed in previous gels.

inin. They may represent cells converting between type 1 and type 2 astrocytes. However, current evidence would suggest this is not the case (Raff and Miller, 1984). A previous study reported that astrocytes from developing brain synthesize laminin in culture (Liesi et al., 1983). While that study also reported a decrease in the synthesis of laminin over time in culture, a similar decrease was not seen in the present study. This disparity may be due to a difference between the 2 studies in the developmental age of the astrocytes or in the cell density of the cultures. The previous study also reported that all astrocytes in culture synthesize laminin. Since the cells in that study were derived from gray matter, an area rich in type 1 astrocytes (Raff et al., 1983a; Miller and Raff, 1984), only type 1 astrocytes may have been examined. It is also possible that type 2 astrocytes from certain brain regions or under certain conditions synthesize laminin.

Laminin immunoreactivity appears to distinguish between type 1 astrocytes in the immature versus the mature optic nerve. During early postnatal development there is a shift in the dominant astrocyte population in the rat optic nerve from type 1 to type 2 astrocytes (Miller et al., 1985). However, type 1 astrocytes reportedly remain in the mature optic nerve (Miller et al., 1985), though these cells, as well as type 2 astrocytes, appear to be laminin-negative. It might be suggested that the ability to synthesize laminin is a step in the differentiation of type 1 astrocytes. Although with Wallerian degeneration of the axons in the optic nerve there is a proliferation of type 1 astrocytes (David et al., 1984), these cells do not express laminin (McLoon, 1986). 
This would suggest that laminin synthesis is not an essential step in the development of all type 1 astrocytes. It seems that the possibility of 2 types of type 1 astrocytes with scparate fates and lineages needs to be considered.

No laminin immunoreactivity is present within the optic nerve after P10, other than that associated with blood vessels. It has been suggested that astrocytes may be involved in the assembly of the basal lamina around vessels of the central nervous system (Lawrence et al., 1984). Therefore, it is possible that astrocytes of the mature optic nerve still synthesize and secrete some laminin. Astrocytes may also contribute laminin to the basal lamina surrounding the optic nerve. The basis for the loss of laminin immunoreactivity around cells within the nerve is not clear. Maturation of the glial population may account for the cessation of laminin synthesis. However, there must also be some mechanism for clearance of laminin after it has been deposited. This may involve proteolysis or perhaps nonproteolytic dissociation of extracellular laminin.

The tcmporal and spatial distribution of laminin immunoreactivity in the developing rat optic stalk and nerve is consistent with the hypothesis that laminin serves as an adhesive substrate for retinal axon growth. Laminin is present in the optic stalk when the first optic axons enter the stalk on E14 (Lund and Bunt, 1976). Furthermore, laminin-positive cells are mostly concentrated in the ventral portion of the stalk, where the first retinal axons grow. $I t$ is tempting to speculate that the distribution of laminin may define the path of growing retinal axons as they traverse the stalk. Our preliminary studies on E18 rats suggest that the distribution of laminin also correlates with the path of optic axons within the brain. Laminin-positive cells are distributed throughout the cross-sectional area of the optic nerve in the late prenatal and early postnatal rat, with the highest concentration at the ventral margin of the nerve. In mammals, growth cones of optic axons appear to be located throughout the cross-sectional area of the nerve, with a higher concentration in the ventral region (Williams and Rakic, 1986; Williams et al., 1986). This contrasts with the developing chick visual system, where growth cones of optic axons are restricted to the most peripheral aspects of the optic nerve (Rager, 1983; McLoon, 1984; Silver and Rutishauser, 1984). It is interesting that laminin immunoreactivity is confined to the area near the external limiting membrane in optic stalk and nerve of the developing chick (McLoon, 1984). In the developing rat optic nerve, the laminin-positive cells were arranged in lines running from the eye towards the brain (Fig. 3). The retinal axon growth cones may have a continuous laminin-containing substrate on which to traverse the nerve by way of these lines of cells. Laminin immunoreactivity appears to be lost from the cells within the rat optic nerve by $P 12$. The last retinal axons grow through the nerve during the first postnatal week (Perry et al., 1983). Thus, laminin disappears from the optic nerve after the last retinal axons have traversed the nerve.

Several things need to be demonstrated in order to more definitively implicate laminin as playing a role in the developmental growth of retinal axons. It needs to be shown that laminin is extracellular in the developing optic nerve, that growth cones are in contact with laminin, and that laminin receptors are on the surface of the growth cones. Live glial cells from developing optic nerve in culture stain with antibodies to laminin where the cell membranes have not been permeabilized by fixative or detergent. This suggests that laminin may be secreted by the cells, at least in vitro. Electron-microscopic immunohistochemistry has shown extracellular laminin within the neuroepithelium of the developing rat brain (Liesi, 1985). In our own preliminary electron-microscopic immunohistochemical study, growth cones have been found in contact with what may be a laminin-positive substrate in the E18 rat optic nerve. However, the density of the staining is less than convincing compared to the heavy laminin staining present in the basal lamina surrounding the nerve. Integrin, a putative laminin receptor, has been identified with the JG22 and CSAT monoclonal antibodies on the surface of chick retinal axons (Cohen et al., 1986; Hall et al., 1987). Though the JG22 antibody blocks the growth of E6 chick ganglion cell neurites on laminin, it has no effect on the growth of these neurites on astrocytes (Cohen et al., 1986). Either the axons respond to some other astrocyte surface molecule, or integrin is not the primary receptor for laminin. A distinct $70 \mathrm{kDa}$ high-affinity receptor for laminin has been identified in other systems (Lesot et al., 1983; Malinoff and Wicha, 1983; Rao et al., 1983). Using light- and electron-microscopic immunohistochemistry, we have shown the transient presence of cranin, a laminin-binding protein that is not related to integrin, on the surface of retinal axons in the developing rat optic nerve (S. C. McLoon and N. R. Smalheiser, unpublished observations). It remains to be determined whether this protein is a laminin receptor.

Laminin may be required for the growth of retinal axons in the mature nervous system as well as during development. Damaged optic axons in the mature mammalian nervous system generally fail to demonstrate more than limited regrowth. Although astrocytes in some regions of the mammalian brain respond to injury with laminin synthesis (Liesi et al., 1983, 1984), no laminin synthesis is induced in the mature optic nerve with optic axon degeneration (McLoon, 1986). Injured adult rat retinal axons will regenerate vigorously into segments of peripheral nerve (So and Aguayo, 1985; Stevenson, 1985), a substrate that does contain laminin (Cornbrooks et al., 1983; Tohyama and Ide, 1984). In species in which retinal axons can regenerate, such as goldfish, regeneration is preceded by the appearance of laminin within the optic nerve (Hopkins et al., 1985). Conditioned medium from neonatal rabbit or regenerating fish optic nerves will elicit a regenerative response from injured retinal axons in maturc mammals (Hadani et al., 1984; Schwartz et al., 1985). This regenerative response is accompanied by an induction of laminin synthesis by cells in the optic nerve (Schwartz et al., 1986). Thus, the early postnatal loss of laminin from the optic nerve may account for the inability of optic axons to regenerate after injury in the mature rat.

The presence of laminin in other areas of the developing brain has also been reported, and it has been suggested that laminin may serve as a substrate for neuronal migration, an event similar in many ways to axon growth (Liesi, 1985; Selak et al., 1985). It is also possible that the production of laminin by astrocytes in the developing brain has a role in neovascularization (Vracko and Benditt, 1972). The appearance of laminin in the optic nerve precedes the arrival of the vasculature, and after vascularization is complete, laminin becomes concentrated around the blood vessels.

The actual role of laminin in the developing nervous system has yet to be resolved. Thus far the evidence is consistent with the idea that laminin serves as an adhesive substrate for growing retinal axons in the developing optic nerve. 


\section{References}

Albrechtsen, R., M. Nielsen, U. Wewer, E. Engvall, and E. Ruoslahti (1981) Basement membrane changes in breast cancer detected by immunohistochemical staining for laminin. Cancer Res. 41: 50765081.

Bignami, A., L. F. Eng, D. Dahl, and C. T. Uyeda (1972) Localization of the glial fibrillary acidic protein in astrocytes by immunofluorescence. Brain Res. 43: 429-435.

Bonhoeffer, F., and J. Huf (1980) Recognition of cell types by axonal growth cones in vitro. Nature 288: 162-164.

Cohen, J., J. F. Burne, J. Winter, and P. Bartlett (1986) Retinal ganglion cells lose response to laminin with maturation. Nature 322 : 465-467.

Cooper, A. R., and H. A. MacQucen (1983) Subunits of laminin are differentially synthesized in mouse eggs and early embryos. Dev. Biol. 96: $467-471$.

Cormbrooks, C. J., D. J. Carey, J. A. McDonald, R. Timpl, and R. P. Bunge (1983) In vivo and in vitro observations on laminin production by Schwann cells. Proc. Natl. Acad. Sci. USA 80: 3850-3854.

Dahl, D., and A. Bignami (1976) Immunogenic properties of the glial fibrillary acidic protein. Brain Res. 116: 150-157.

David, S., R. H. Miller, R. Patel, and M. C. Raff (1984) Effects of neonatal transection on glial cell development in the rat optic nerve: Evidence that the oligodendrocyte-type 2 astrocyte cell lineage depends on axons for its survival. J. Neurocytol. 13: 961-974.

Davis, G. E., M. Manthorpe, E. Engvall, and S. Varon (1985) Isolation and characterization of rat Schwannoma neurite-promoting factor: Evidence that the factor contains laminin. J. Neurosci. 5: 2662-2671.

Eisenbarth, G. S., F. S. Walsh, and M. Nirenberg (1979) Monoclonal antibody to a plasma membrane antigen of neurons. Proc. Natl. Acad. Sci. USA 76: 4913-4917.

Hadani, M., A. Harel, A. Solomon, M. Belkin, V. Lavie, and M. Schwartz (1984) Substances originating from the optic nerve of neonatal rabbit induce regeneration-associated response in the injured optic nerve of adult rabbit. Proc. Natl. Acad. Sci. USA 81: 7965-7969.

Hall, D. E., K. M. Neugebauer, and L. F. Reichardt (1987) Embryonic neural retinal cell response to extracellular matrix proteins: Developmental changes and effects of the CSAT antibody. J. Cell Biol. 104: 623-633.

Hammarback, J. A., S. L. Palm, L. T. Furcht, and P. C. Letourneau (1985) Guidance of neurite outgrowth by pathways of substratumadsorbed laminin. J. Neurosci. Res. 13: 213-220.

Hopkins, J. M., T. S. Ford-Holevinski, J. P. McCoy, and B. W. Agranoff (1985) Laminin and optic nerve regeneration in the goldfish. J. Neurosci. 5: 3030-3038.

Kuhl, V., R. Timpl, and K. von der Mark (1982) Synthesis of type IV collagen and laminin in cultures of skeletal muscle cells and their assembly on the surface of myotubes. Dev. Biol. 93: 344-354.

Laemmli, U. K. (1970) Cleavage of structural proteins during the assembly of the head of bacteriophage T4. Nature 227: 680-684.

Lander, A. D., D. K. Fujii, and L. F. Reichardt (1985) Laminin is associated with the "neurite outgrowth-promoting factors" found in conditioned media. Proc. Natl. Acad. Sci. USA 82: 2183-2187.

Lawrence, J. M., S. K. Huang, and G. Raisman (1984) Vascular and astrocytic reactions during establishment of hippocampal transplants in adult host brain. Neuroscience 12: 745-760.

Lesot, H., U. Kuhl, and K. von der Mark (1983) Isolation of a lamininbinding protein from muscle cell membrane. EMBO J. 2: 861-865.

Letoumeau, P. C. (1975a) Possible roles for cell-to-substratum adhesion in neuronal morphogenesis. Dev. Biol. 44: 77-91.

Letourneau, P. C. (1975b) Cell-to-substratum adhesion and guidance of axonal elongation. Dev. Biol. 44: 92-101.

Liesi, P. (1985) Do neurons in the vertebrate CNS migrate on laminin? EMBO J. 4: 1163-1170.

Liesi, P., D. Dahl, and A. Vaheri (1983) Laminin is produced by early rat astrocytes in primary culture. J. Cell Biol. 96: 920-924.

Liesi, P., S. Kaakkola, D. Dahl, and A. Vaheri (1984) Laminin is induced in adult brain astrocytes by stereotaxic lesioning. EMBO J. 3: $683-686$.

Lund, R. D., and A. H. Bunt (1976) Prenatal development of central optic pathways in albino rats. J. Comp. Neurol. 165: 247-264.

Malinoff, H. L., and M. S. Wicha (1983) Isolation of a cell surface receptor protein for laminin from murine fibrosarcoma cells. J. Cell Biol. 96: 1475-1479.
Manthorpe, M., E. Engvall, E. Ruoslahti, F. M. Longo, G. E. Davis, and S. Varon (1983) Laminin promotes neuritic regeneration from cultured peripheral and central neurons. J. Cell Biol. 97: 1882-1890.

McLoon, L. K., and S. C. McLoon (1988) Schwann cell conditioned medium promotes neurite outgrowth from explants of fetal rat retina and tectum in vitro. Dev. Brain Res. 39: 61-68.

McLoon, S. C. (1984) Distribution of laminin in the developing visual system of the chick. Soc. Neurosci. Abstr. 10: 466.

McLoon, S. C. (1986) Response of astrocytes in the visual system to Wallerian degeneration: An immunohistochemical analysis of laminin and GFAP. Exp. Neurol. 91: 613-621.

Miller, R. H., and M. C. Raff (1984) Fibrous and protoplasmic astrocytes are biochemically and developmentally distinct. J. Neurosci. 4: 585-592.

Miller, R. II., S. David, R. Patel, E. R. Abney, and M. C. Raff (1985) A quantitative immunohistochemical study of macroglial cell development in the rat optic nerve: In vivo evidence for two distinct astrocyte lineages. Dev. Biol. 111: 35-41.

Noble, M., J. Fok-Seang, and J. Cohen (1984) Glia are a unique substrate for the in vitro growth of central nervous system neurons. J. Neurosci. 4: 1892-1903.

Palm, S. L., and L. T. Furcht (1983) Production of laminin and fibronectin by Schwannoma cells: Cell-protein interactions in vitro and protein localization in peripheral nerve in vivo. J. Cell Biol. 96: 12181226.

Perry, V. H., Z. Henderson, and R. Linden (1983) Postnatal changes in retinal ganglion cell and optic axon populations in the pigmented rat. J. Comp. Neurol. 219: 356-368.

Raff, M. C., and R. H. Miller (1984) Glial cell development in the rat optic nerve. Trends Neurosci. 7: 469-472.

Raff, M. C., E. R. Abney, J. Cohen, R. Lindsay, and M. Noble (1983a) Two types of astrocytes in cultures of developing rat white matter: Differences in morphology, surface gangliosides, and growth characteristics. J. Neurosci. 3: 1289-1300.

Raff, M. C., R. H. Miller, and M. Noble (1983b) A glial progenitor cell that develops into an astrocyte or an oligodendrocyte depending on culture medium. Nature 303: 390-396.

Rager, G. (1983) Structural analysis of fiber organization during development. Prog. Brain Res. 58: 313-319.

Rao, N. C., S. H. Barsky, V. P. Terranova, and L. A. Liotta (1983) Isolation of a tumor cell laminin receptor. Biochem. Biophys. Res. Commun. 111: 804-808.

Rogers, S. L., P. C. Letourneau, S. L. Palm, J. McCarthy, and L. T. Furcht (1983) Neurite extension by peripheral and central nervous system neurons in response to substratum-bound fibronectin and laminin. Dev. Biol. 98: 212-220.

Rogers, S. L., K. J. Edson, P. C. Letourneau, and S. C. McLoon (1986) Distribution of laminin in the developing peripheral nervous system of the chick. Dev. Biol. 113: 429-435.

Sakashita, S., E. Engvall, and E. Ruoslahti (1980) Basement membrane glycoprotein laminin binds to heparin. FEBS Lett. 116: 243-246.

Sanes, J. R. (1983) Role of extracellular matrix in neural development. Annu. Rev. Physiol. 45: 581-600.

Schachner, M., E. T. Hedley-Whyte, D. W. Hsu, G. Schoonmaker, and A. Bignami (1977) Ultrastructural localization of glial fibrillary acidic protein in mouse cerebellum by immunoperoxidase labeling. J. Cell Biol. 75: 67-73.

Schwartz, M., M. Belkin, A. Harel, A. Solomon, V. Lavie, M. Hadani, I. Rachailovich, and C. Stein-Izsak (1985) Regenerating fish optic nerves and a regeneration-like response in injured optic nerves of adult rabbits. Science 228: 600-603.

Schwartz, M., A. Harcl, N. Zak, Y. Bawnik, C. Stcin-Izsak, and Z. Vogcl (1986) Regeneration-associated environmental changes are induced in the injured optic nerve of adult rabbit. Soc. Neurosci. Abstr. 12: 12.

Selak, I., J. M. Foidart, and G. Moonen (1985) Laminin promotes cerebellar granule cell migration in vitro and is synthesized by cultured astrocytes. Dev. Neurosci. 7: 278-285.

Silver, J., and U. Rutishauser (1984) Guidance of optic axons in vivo by a preformed adhesive pathway on neuroepithelial endfeet. Dev. Biol. 106: 485-499.

Smalheiser, N. R., S. M. Crain, and L. M. Reid (1984) Laminin as a substrate for retinal axons in vitro. Dev. Brain Res. 12: 136-140.

So, K.-F., and A. J. Aguayo (1985) Lengthy regrowth of cut axons 
from ganglion cells after peripheral nerve transplantation into the retina of adult rats. Brain Res. 328: 349-354.

Stevenson, J. A. (1985) Growth of optic tract axons in nerve grafts in hamsters. Exp. Neurol. 87: 446-457.

Terranova, V. P., C. N. Rao, T. Kalebic, I. M. Margulies, and L. A. Liotta (1983) Laminin receptor on human breast carcinoma cells. Proc. Natl. Acad. Sci. USA 80: 444-448.

Timpl, R., H. Rohde, P. G. Robey, S. I. Rennard, J.-M. Foidart, and G. R. Martin (1979) Laminin - a glycoprotein from basement membrane. J. Biol. Chem. 254: 9933-9937.

Tohyama, K., and C. Ide (1984) The localization of laminin and fibronectin on the Schwann cell basal lamina. Arch. Histol. Jpn. 47: 519-532.

Towbin, H., T. Staehelin, and J. Gordon (1979) Electrophoretic transfer of proteins from polyacrylamide gels to nitrocellulose sheets: Procedure and some applications. Proc. Natl. Acad. Sci. USA 76: 43504354 .
Tramu, G., A. Pillez, and J. Leonardelli (1978) An efficient method of antibody elution for the successive or simultaneous localization of two antigens by immunocytochemistry. J. Histochem. Cytochem. 26: 322-324.

Vracko, R., and E. P. Benditt (1972) Basal lamina: The scaffold for orderly cell replacement. Observations on regeneration of injured skeletal muscle fibers and capillaries. J. Cell Biol. 55: 406-419.

Williams, R. W., and P. Rakic (1986) Dispersion of growing axons within the optic nerve of the embryonic monkey. Proc. Natl. Acad. Sci. USA 82: 3906-3910.

Williams, R. W., M. J. Bastiani, B. Lia, and L. M. Chalupa (1986) Growth cones, dying axons and developmental fluctuations in the fiber population of the cat's optic nerve. J. Comp. Neurol. 246: 3269. 